\title{
Numerical Simulation of Rotor in Hover and Forward Flight
}

\author{
Qinghe Zhao ${ }^{\text {a }}$ \\ School of Energy and Power Engineering, Beihang University, Beijing 100191, China
}

\begin{abstract}
The flow around rotor is numerical simulated in hover and forward flight based on multi-structured grid. In hover the flow field can be transformed into a steady-state flow field in the rotating coordinate system. The experimental data of Caradonna and Tung rotor is used to verify the numerical simulation result. The numerical results compare well with the experimental data for both non-lifting and lifting cases. Non-lifting forward flight is simulated and the prediction capabilities have been validated through the ONERA two-blade rotor. The pressure distributions of different positions under different azimuth angles are compared, which is in good agreement with the experimental data. There is unsteady shock wave when forward flight. Dual-time method is used to obtain unsteady flow field with rigid moving grid in the inertial system.
\end{abstract}

\section{INTRODUCTION}

The flow around rotor blade has many different characteristics from the fixed wing. The local velocity of rotor varies linearly with the radius of rotation. Across the tip of the transonic speed and near the hub of the incompressible flow area often occur at the same time. Furthermore, the induced velocity produced by the rotor wake vortex system has an important influence on the flow field. The flow field is unsteady and characterized by strong non-linear and three-dimensional effects. Transonic regions near the advancing blade tip leading to shock-induced separation, as well as extensive regions of separated flow due to dynamic stall occurring on retreating blades.

For the hover state, the coordinate system is established on the blade and rotates together with the rotor. The stationary flow field with rotational symmetry is observed. In the rotational coordinate system to solve the equation can greatly improve the hover flow field calculation efficiency. Unlike in hover, the forward flight involves asymmetric flow fields and cannot be solved in a rotating coordinate system. It should be simulated unsteady flow field, which require more computational resource.

A large number work had been carried out to solve flow field in hover with structural meshes [1,2]. High order scheme such as ENO is used to distinguish the flow characteristics [3]. The Chimera method was used to solve the flow field. The blade-fixed grids are embedded in the background grid. Along the chimera boundaries, a tri-linear interpolation method is used to couple the solution of the blade-fixed and background grids. Unstructured grid is used to refine the flow field [4]. Adaptive mesh refinement involves automatically refining the mesh to resolve important flow characteristics. A detailed overview was introduced on the hovering rotor [5]. in hover simulation [6]. Multi-block

\footnotetext{
${ }^{a}$ Corresponding author : zhaoqinghe2001@163.com
} 
structured grid combined deforming mesh method is used to simulate the forward flight flow field [7]. Blade-vortex interactions acoustic is introduced [8,9]. Forward flight filed is simulated [10]. Simulation progress in Europe is introduced [11]. Despite these methods, multi-block structured grids are still the most commonly used form and are widely used in solving rotor flow field.

In the present work, multi-block structured grids are used. The structure of the paper is as follows. Section 3 presents the governing equations in an rotational coordinate system, as used in the hovering simulations. The spatial discretization and temporal integration method are described, followed by the forward flight in a inertial frame of reference. Validation results are presented in Section 3 for both hovering and forward flight cases. In this paper, the work of Caradonna and Tung, the experiments of ONERA were used. Conclusions are finally drawn in Section 4.

\section{NUMERICAL SOLUTION PROCEDURES}

For hover cases, the flow around a hovering rotor can be treated as a steady problem. For forward flight simulations the flow field is asymmetry and unsteady. So the simulation process is introduced separately.

\subsection{Methodologies for hover case}

For hover cases, the flow around a hovering rotor can be treated as a steady problem. For forward flight simulations the flow field is asymmetry and unsteady. So the simulation process is introduced separately.

Due to the symmetry of the flow in rotational coordinate system, The computational domain can be replaced only by a domain around one blade. The periodicity boundary condition is used to taken into the influence of other blades. Therefore, the computational expense can be reduced greatly. The government equation is given below

$$
\frac{d}{d t} \int_{V(t)} \stackrel{\stackrel{\mathrm{r}}{w}}{w} d V+\int_{\partial V(t)}\left(\stackrel{\mathrm{r}}{F}(\stackrel{\mathrm{r}}{w})-\stackrel{\mathrm{r}}{F_{v}}(\stackrel{\mathrm{r}}{w})\right) \stackrel{\mathrm{r}}{\mathrm{r}} d S=\stackrel{\mathrm{r}}{S}
$$

Where $\stackrel{1}{w}$ is the conserved variables. $\stackrel{1}{F}$ and $\stackrel{1}{F}$ are the inviscid and viscous fluxes, respectively. Two-equation $k-\omega$ turbulence model is used to simulate the viscous flow field. There is a source terms in the non-inertial frame of reference. It means the influence of the centripetal and the Coriolis force. For an hovering rotor rotating around the y-axis with the angular velocity $\stackrel{\mathrm{r}}{\omega}=[0, \Omega, 0]$, the source term $\stackrel{1}{S}$ can be expressed by

$$
\underset{w}{\mathrm{r}}=\left[\begin{array}{c}
\rho \\
\rho u \\
\rho v \\
\rho w \\
\rho E
\end{array}\right], \quad \stackrel{\mathrm{r}}{F}=\left[\begin{array}{c}
\rho\left(q-q_{\omega}\right) \\
\rho u\left(q-q_{\omega}\right)+p i_{x} \\
\rho v\left(q-q_{\omega}\right)+p i_{y} \\
\rho w\left(q-q_{\omega}\right)+p i_{z} \\
\rho h\left(q-q_{\omega}\right)+p q_{\omega}
\end{array}\right], \quad \stackrel{\mathrm{r}}{S}=\left[\begin{array}{c}
0 \\
-\rho \Omega w \\
0 \\
\rho \Omega u \\
0
\end{array}\right]
$$

Where $q_{\omega}$ is the grid velocity, it produce a grid flux. The finite volume discretization with central differencing for the flux approximation leads to 2 order scheme. The implicit Lower-Upper Symmetric Gauss Seidel operator(LUSGS) is applied to obtain the update solution.

\subsection{Methodologies for unsteady flows}


For forward flight, the flow field should be simulated in unsteady condition. The dual time stepping approach is introduced, and the system can be solved by an iteration through pseudo time to the steady state. The implicit dual-time approach introduced by Jameson has become a very attractive method for solving such problems as it allows an implicit discretisation to be used in real time. The steady state is reached at a pseudo time in each time step.

$$
\begin{gathered}
\frac{3 V_{i, j, k}^{n+1} w_{i, j, k}^{n+1}-4 V_{i, j, k}^{n} w_{i, j, k}^{n}+V_{i, j, k}^{n-1} w_{i, j, k}^{n-1}}{2 \Delta t}+R_{i, j, k}\left(w_{i, j, k}^{n+1}\right)=0 \\
V_{i, j, k}^{n+1} \frac{w_{i, j, k}^{n+1, m+1}-w_{i, j, k}^{n+1, m}}{\Delta \tau}+\frac{3 V_{i, j, k}^{n+1} w_{i, j, k}^{n+1, m}-4 V_{i, j, k}^{n} w_{i, j, k}^{n}+V_{i, j, k}^{n-1} w_{i, j, k}^{n-1}}{2 \Delta t}+R_{i, j, k}\left(w_{i, j, k}^{n+1, m}\right)=0 \\
V_{i, j, k}^{n+1}\left(\frac{1}{\Delta \tau}+\frac{3}{2 \Delta t}+A+B+C\right) \Delta w_{i, j, k}=-R_{i, j, k}\left(w_{i, j, k}^{n+1, m}\right)-\frac{3 V_{i, j, k}^{n+1} w_{i, j, k}^{n+1, m}-4 V_{i, j, k}^{n} w_{i, j, k}^{n}+V_{i, j, k}^{n-1} w_{i, j, k}^{n-1}}{2 \Delta t}
\end{gathered}
$$

Where $\Delta w_{i, j, k}=w_{i, j, k}^{n+1, m+1}-w_{i, j, k}^{n+1, m}, \Delta t$ is the physical time step, $\Delta \tau$ is the pseudo time step. $A, B, C$ is the Jacobi metrics of the inviscid flux. The accelerate convergence methods such as local time step can be used in the pseudo time cycles.

\subsection{Mesh movement}

There is no change of the relative position of points in the same grids if the rotor moves rigidly. Assuming it rotate around the $y$-axis, the transformation matrices are introduced for rotation. And the grid coordinate and grid velocity can be calculated directly from formula 6 . Where 0 denotes the original grid position and $\mathrm{n}$ denotes the updated grid position. The mesh velocity introduced is essentially the mesh rotation velocity.

$$
\left[\begin{array}{l}
x \\
y \\
z
\end{array}\right]^{n}=\left[\begin{array}{ccc}
\cos \omega t & 0 & \sin \omega t \\
0 & 1 & 0 \\
-\sin \omega t & 0 & \cos \omega t
\end{array}\right]\left[\begin{array}{l}
x \\
y \\
z
\end{array}\right]^{0} \quad\left[\begin{array}{l}
u \\
v \\
w
\end{array}\right]^{n}=-\omega\left[\begin{array}{ccc}
\sin \omega t & 0 & -\cos \omega t \\
0 & 0 & 0 \\
\cos \omega t & 0 & \sin \omega t
\end{array}\right]\left[\begin{array}{l}
x \\
y \\
z
\end{array}\right]^{0}
$$

There is no grid volume changing, so the geometric conservation law(GCL) is also kept.

\section{THE HOVER CASE}

An experimental study of a helicopter model in hover had been carried out by Caradonna and Tung in 1981 [12]. This experimental data is usually used to verify the CFD program. The blade had a NACA0012 profile, which had a diameter of 2.286 meter, and a chord length of $0.191 \mathrm{~m}$, according to the aspect ratio was 6 . In addition, since the flow field is symmetric, only one blade can be modelled by periodic boundary conditions. Figure 1 shows the grid topology. The rotor rotation centre is on the $\mathrm{z}$-axis, the rotor blades are on the $\mathrm{y}$-axis, a quarter of the point is at $\mathrm{x}=0$ and the leading edge of the blade points to the positive $y$-axis. The rotor hub is a simple straight cylinder. In the calculation, flow field is initialled by static flow conditions.

In hover the pressure coefficient is calculated by formula 7, where $\omega$ is the rotate velocity and $r$ is the span distance.

$$
C p=\frac{p-p_{0}}{0.5 \rho(\omega r)^{2}}
$$



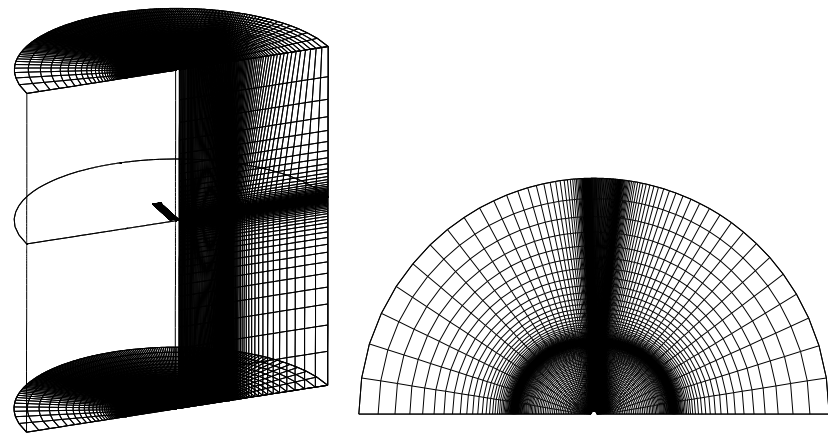

Figure 1. Grid topology of Caradonna and Tung

\subsection{Non-lifting in hover}

The non-lifting in hover case is simulated firstly. For this case the tip Mach number is 0.52 and the corresponding Reynolds number is 2.32 million. Figure 2 shows the comparison of CFD results to the experimental data for the pressure coefficient distribution at five different sections $(\mathrm{r} / \mathrm{R}=0.50,0.68,0.80,0.89,0.96)$. The comparison shows a good agreement with the experimental data. At the same time the pressure contour is given, it can be seen that there is a low pressure zone at the rotor tip.
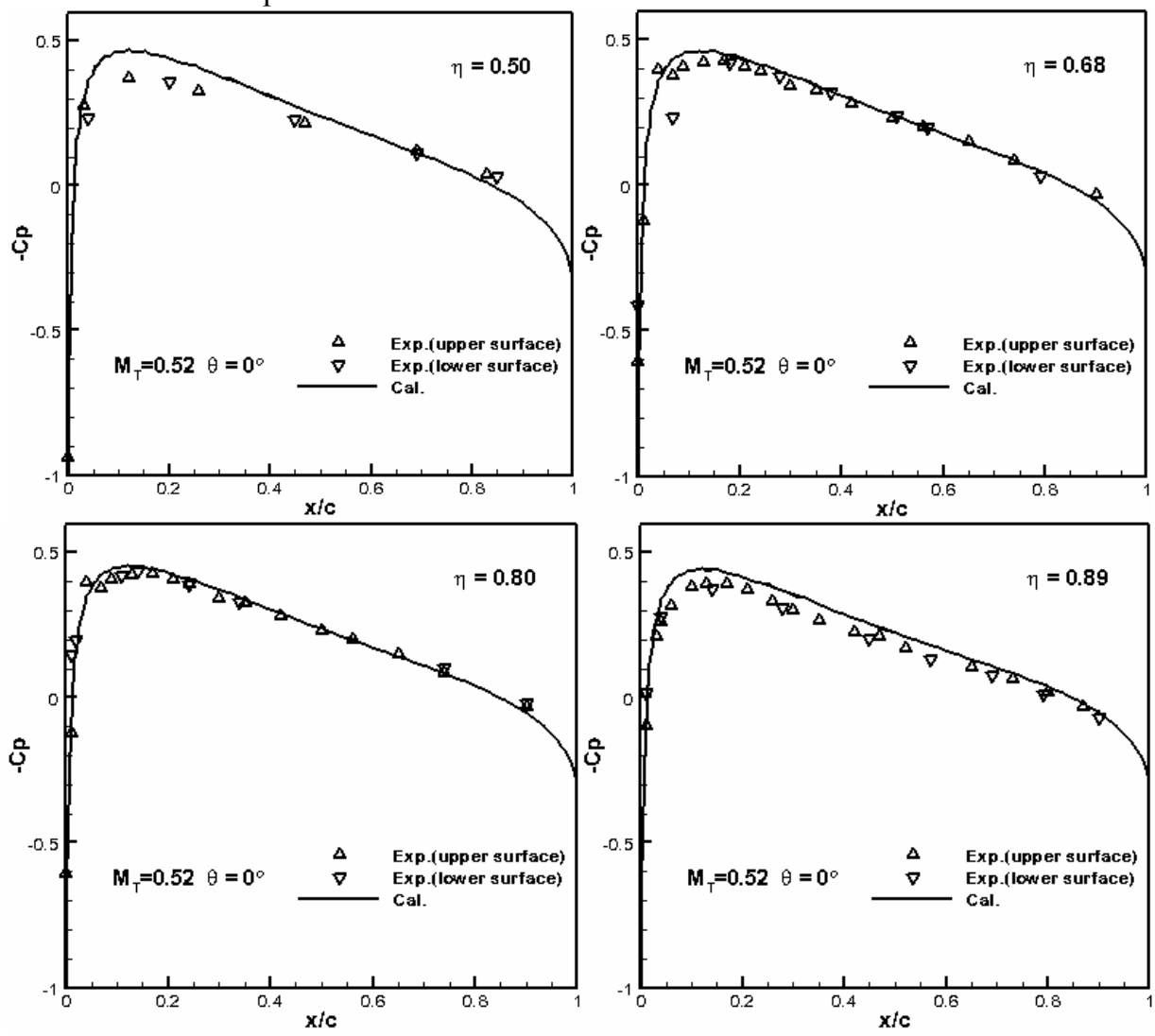

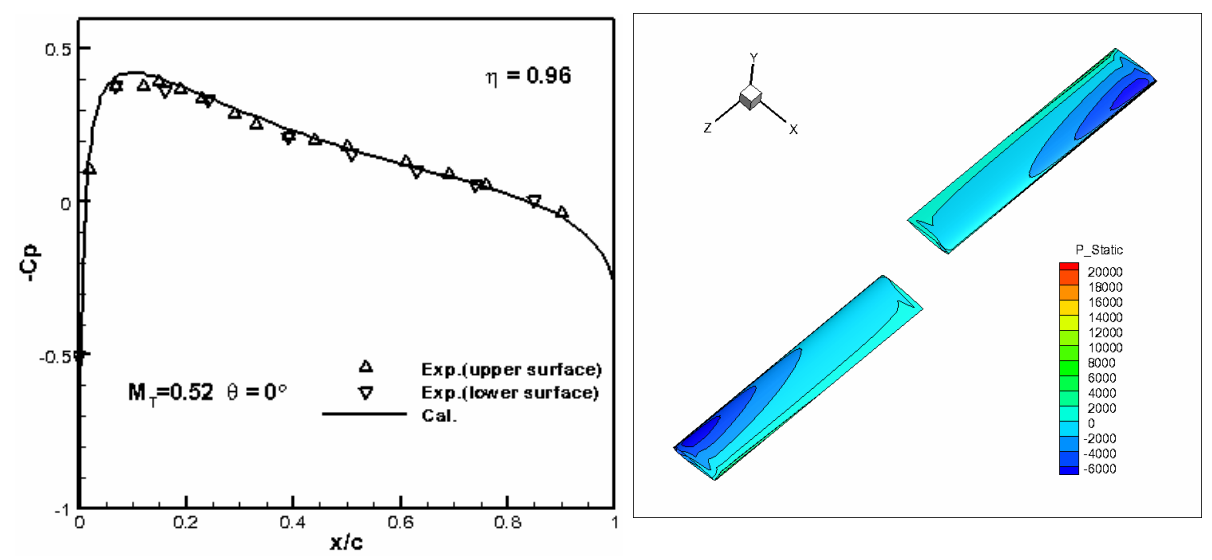

Figure 2. Pressure distribution comparison of flow field with non-lifting in hover

\subsection{Lifting in hover}

For transonic lifting in hover, the tip Mach number is 0.877 , the total angle is $8^{\circ}$ and the corresponding Reynolds number is 3.93 million. Figure 3 shows the comparison of pressure coefficient calculated with the experimental data at different sections, except that the calculated pressure coefficient on the first section is higher than the experimental values, and the calculated values of the other sections are generally in good agreement with the experimental values. From the pressure contour it can be seen that the supercritical flow conditions was dominated by stronger transonic shocks in the rotor tip region.
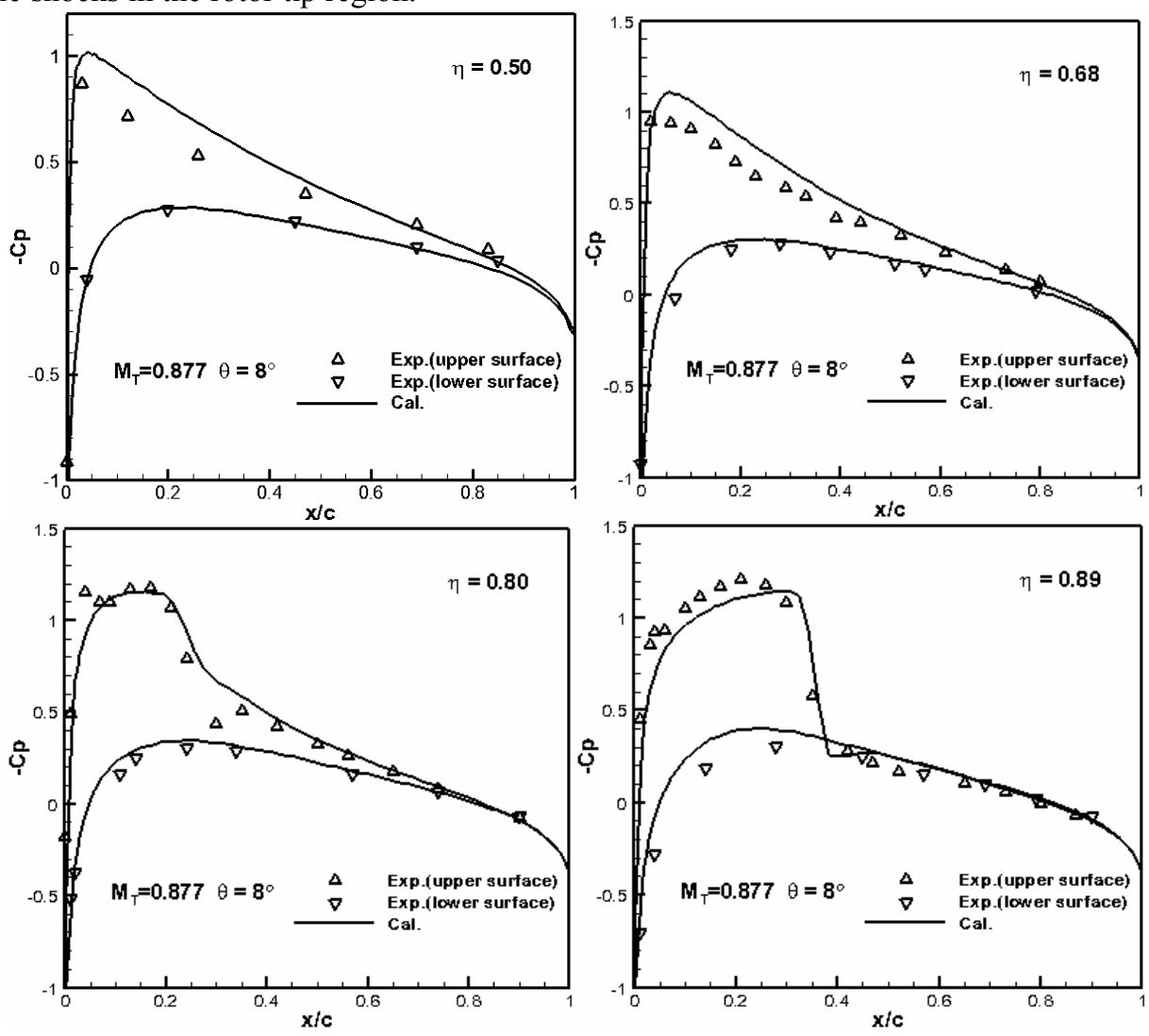

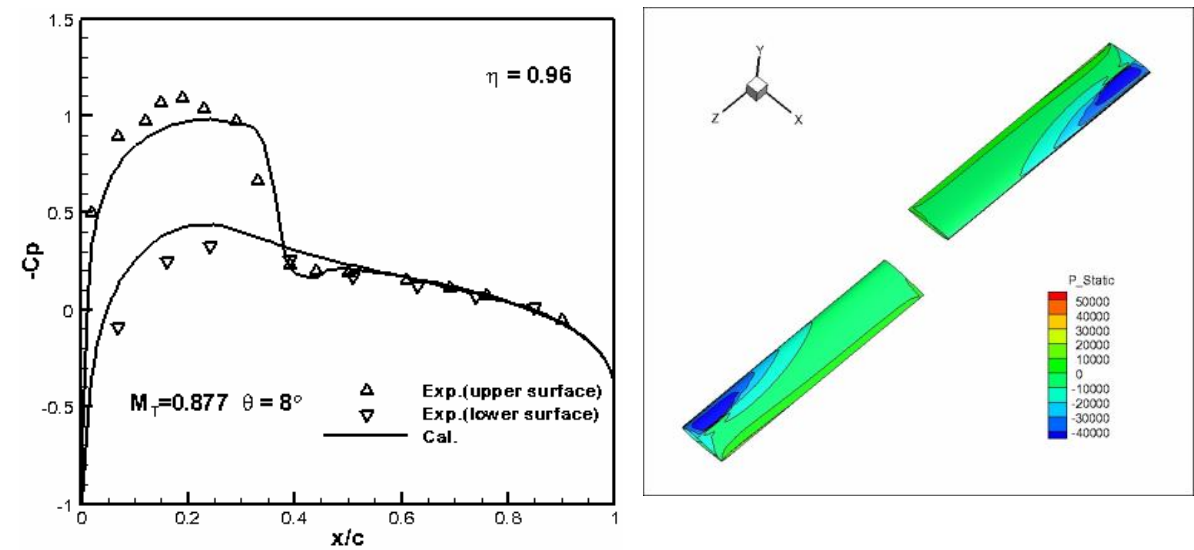

Figure 3. Pressure distribution comparison of flow field with lifting in hover

\section{FORWARD FLIGHT CASE}

This section presents the results for ONERA two-blade rotor in non-lifting forward flight [13]. The flight condition is advance ratio 0.5 and a tip Mach number 0.625 . The profile of the rotor changes from NACA0017 at the root to NACA0009 at the tip. Since the forward flow field is unsteady, the entire grid is used to solve the field. The Figure 4 shows the topology of the grid.
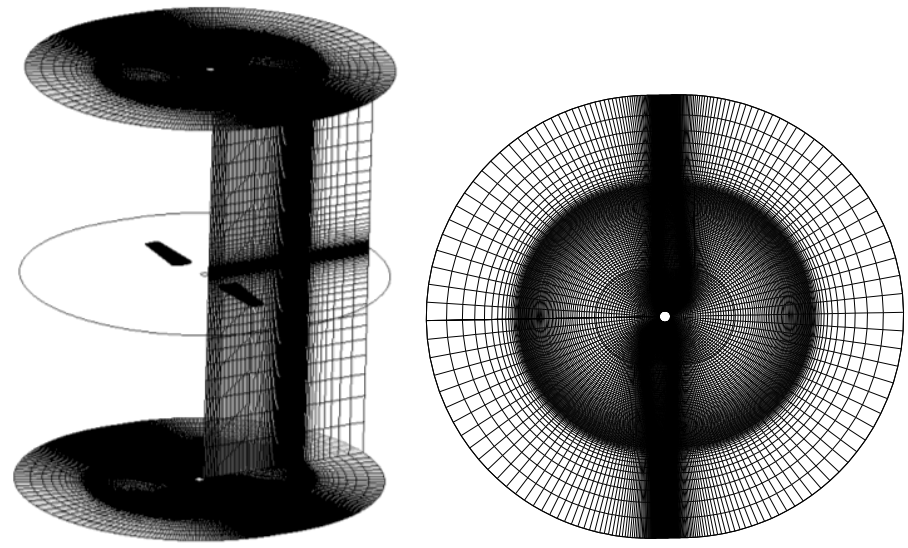

Figure 4. Grid topology of ONERA rotor

The pressure coefficient calculation of forward flight depend on not only the local rotation speed, but also forward flight speed, the formula is given by formula 8

$$
C p=\frac{p-p_{0}}{0.5 \rho\left(\omega r+V_{\infty} \cdot \sin \varphi\right)^{2}}
$$

The simulation results have been compared with the experimental data. The azimuthal step used for forward flight is $\Delta \psi=0.25^{\circ}$, which is equivalent to a rotational period includes 1440 time steps, 50 inner iterations is used in each time step. The figure 5 shows the pressure contour of several azimuths. Respectively $0,30,60,90,120,150,180,210,240,270,300,330$ degree. It can be seen that there is a clear shock wave when forward flight. The shock wave diminishes or even disappears when the blade is moving backward. 


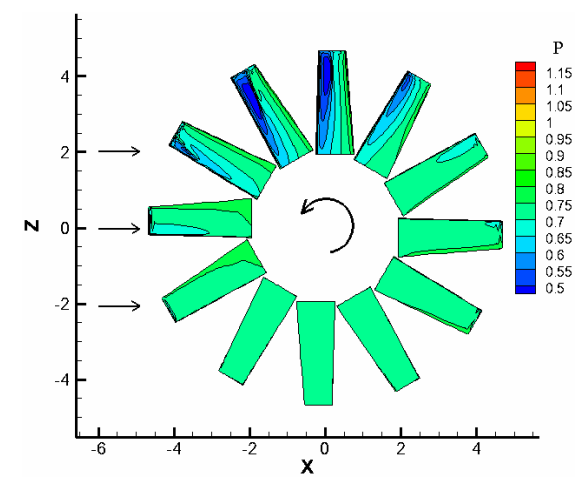

Figure 5. Pressure contour of the ONERA rotor forward flight

Figure 6 shows the comparison of the pressure distribution at 0.85 and 0.90 section with experimental values at different azimuths. The experimental data corresponds to the forward flight condition. There is no experiment data for the retreating side. The present method compares quite well with the experimental data for the azimuthal positions of $\psi=60,90,120$. The biggest differences is encountered at $\psi=150^{\circ}$. At this azimuch the flow is highly unsteady and the shock is moving rapidly downstream.
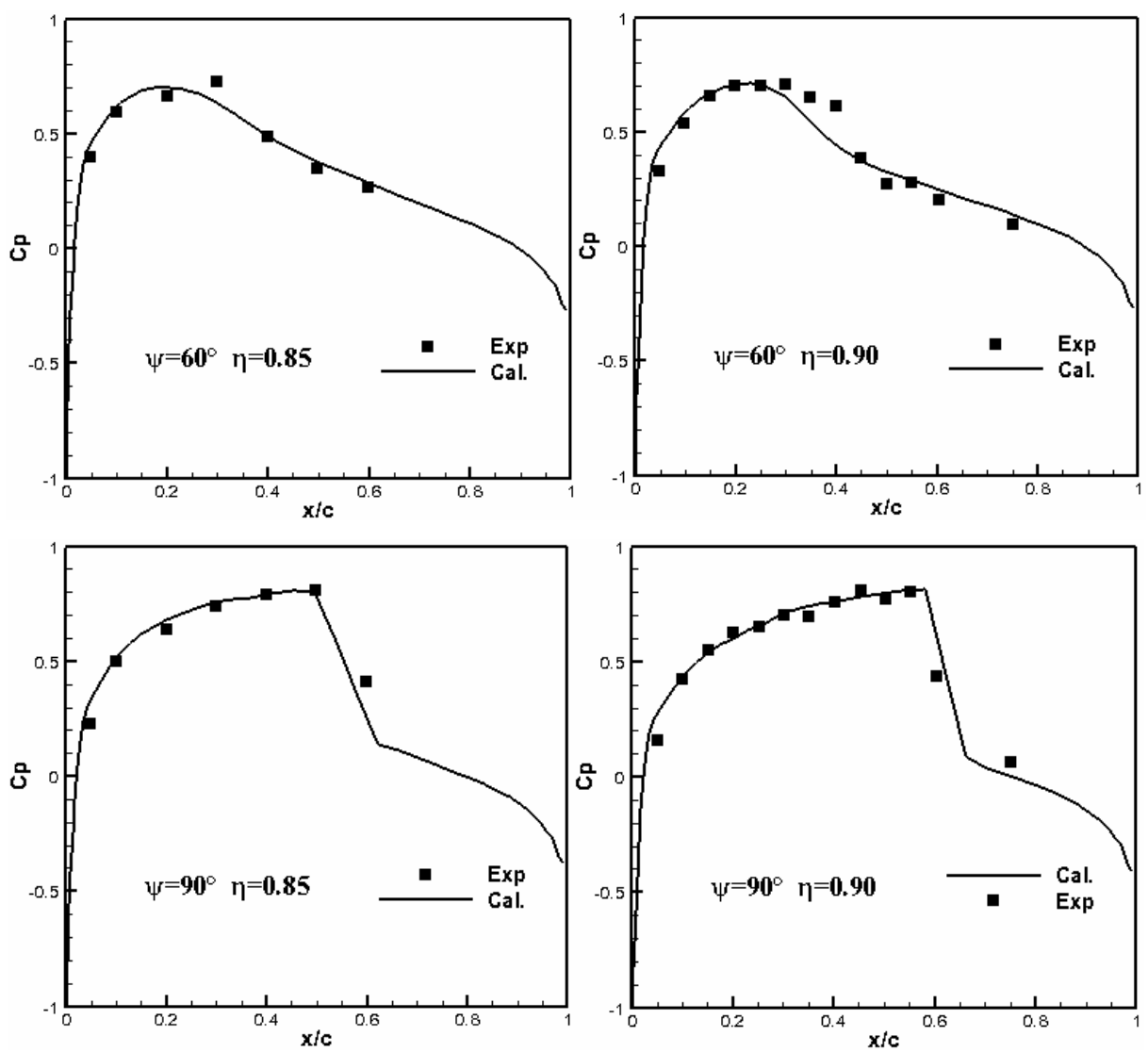

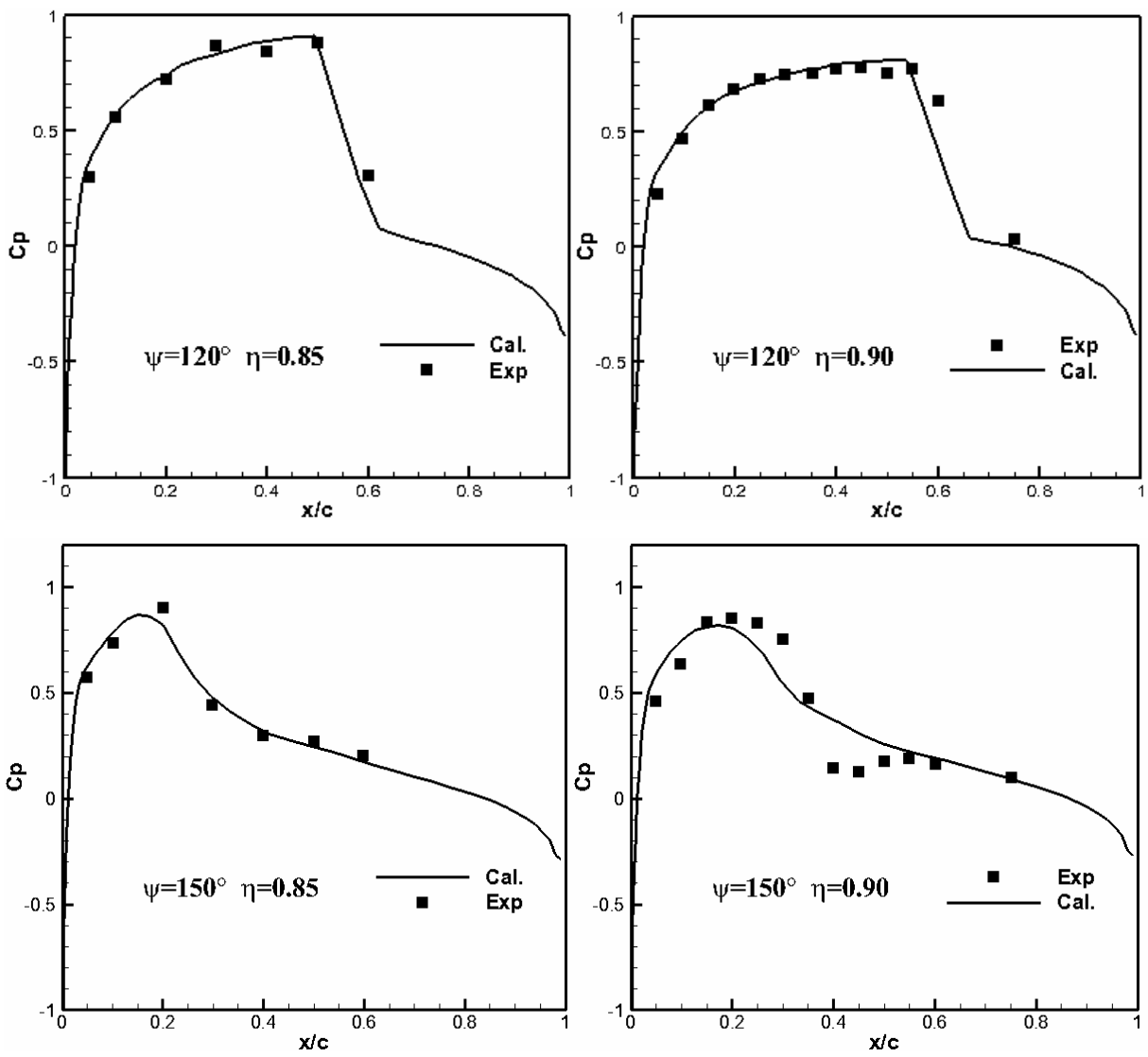

Figure 6. Chord-wise $\mathrm{Cp}$ distribution for the ONERA rotor at two radial stations

\section{CONCLUSION}

A numerical approach is presented for the simulation of hovering and forward flight flow field based on multi-block grid. In hover the flow field can be transformed into a steady-state in the rotating coordinate system. The numerical results compare very well with the experimental data for both nonlifting and lifting cases. Forward flight prediction capabilities have been validated through the ONERA two-blade rotor. The pressure distributions at different positions under different azimuth angles are compared, which is in good agreement with the experimental data.

\section{References}

1. Srinivasan, G. R., and McCroskey, W. J. (1988) Navier-Stokes Calculations of Hovering Rotor Flowfields AIAA Journal Vol 25 No 10

2. Srinivasan, G. R., and Baeder, J. D. (1992) Flowfield of lifting rotor in hover: A Navier-Stokes simulation AIAA Journal Vol 30 No 10

3. Hariharan, N., and Sankar, L. (2004) High-Order Essentially Non-oscillatory Schemes for Rotary-Wing Wake Computations, Journal of Aircraft Vol 41 No 2, 2004

4. Strawn,R.C., and Barth,T.J. (1993) A finite-volume Euler Solver for computing Rotary-Wing Aerodynamics on Unstructured Meshes Journal of American Helicopter Society Vol 38,(2) pp.61-67

5. Hariharan, N., Egolf, A., and Sankar, L. (2014) Simulation of Rotor in Hover: Current state and challenges AIAA paper 2014-0041 
6. Pomin, H., and Wagner, S. (2002) Navier-Stokes analysis of isolated rotor flow in helicopter hover flight Journal of Aircraft 39(5) 813-821

7. Steijl, R., Barakos, G., and Badcock, K. (2006) A framework for CFD analysis of helicopter rotors in hover and forward flight International Journal for numerical methods in fluids 51:819-847

8. Yung, H.Yu., Chee, Tung., Judith, Gallman., Klaus J, Schultz., Berend, der. Wall., Pierre, Spiegel., and Bertrand, Michea. (1995) Aerodynamics and Acoustic of Rotor Blade-Vortex Interactions, Journal of Aircraft Vol 32 No 5

9. Lyaysan, Ildusovna. Garipova., Andrei, Sergeevich. Batrakov., Alexander, Nikolaevich. Kusyumov., and Sergey, Anatolievich. Mikhaylov. (2016) Aerodynamic and acoustic analysis of helicopter main rotor blade tips in hover, International Journal of Numerical Methods for Heat \& Fluid Flow Vol.26 No.7

10. Yang,Z., Sankar,L.N., Smith,M.J., and Bauchau,O. (2002) Recent Improvements to a Hybrid Method for Rotors in Forward Flight, Journal of Aircraft Vol 39 No 5

11. Renzoli, P. D'Alascio., A. Kroll., N. Peshkin. D., and H,L.Hounjet. M. (2000) EROS-a common European Euler code for the analysis of the helicopter rotor flowfield Progress in Aerospace Sciences 36:437-485

12. Caradonna, F. X., and Tung, C. (1981). Experimental and analytical studies of a model helicopter rotor in hover Vertica 5(1):149-161

13. Philippe J-J, Chattot J-J. (1980) Experimental and theoretical studies on helicopter blade tips at ONERA. Sixth European Rotorcraft and Powered Lift Aircraft Forum, Bristol, September 